\title{
Polyoma BK virus infection in renal transplant recipients
}

Nicola liritano, Maria Gabriella Lepore, Vincenzo Rondinelli, Vittorio Focarelli, Pasquale Minchella, Raffaele Saraceno, Stefania Giglio, Paolo Opipari, Sandra Castagna, Rosanna Masciari

Virologia e Microbiologia Azienda Ospedaliera Pugliese-Ciaccio, Catanzaro

Key words: Polyomavirus BK, interstitial nephritis, immunosoppressive treatment, viral load

Infezione da Polyoma BK virus (BKV) dopo trapianto di rene

\section{SUMMARY}

Several studies reported a correlation between the human Polyomavirus BK (BKV) and interstitial nephritis (PVN: Polyoma Virus Nephropaty) in renal transplant recipients in whom immunosuppressive treatment is thought to facilitate or induce reactivation of the virus. In the present study we monthly evaluated the presence of BKV-DNA in plasma and urine of 29 kidney transplant recipients.

We used a nested PCR for BKV-DNA screening in plasma and urine and a quantitative assay Real Time PCR in case of a positive screening result.

The viral DNA has been detected in $48 \%$ of the patients samples: only in urine of six patients; in plasma of four and in both of two. Immunosuppressive therapy has been decreased in positive patients.

The kidney loss occurred just in the two patients with high viral load in plasma and urine.

The definitive diagnosis of BKV Nephropaty requires allograft biopsy though biomolecular test for BKV-DNA in urine (viruria) and in plasma (viremia) could be very important non-invasive method for the early diagnosis of PVN.

\section{INTRODUZIONE}

Il virus BK (BKV) è un Polyomavirus umano (40 nm) della famiglia Polyomaviridae con DNA a doppio filamento. I virioni hanno forma sferica, sono privi di envelope e con capside icosaedrico. BKV utilizza per la sua replicazione la polimerasi della cellula ospite e codifica per 5 proteine di cui 3 capsidiche (VP1, VP2, VP3).

Diversi studi hanno dimostrato che più del $70 \%$ della popolazione adulta possiede anticorpi per BKV, avendo contratto, per via respiratoria, un'infezione primaria durante l'infanzia, di solito nei primi 3-4 anni di età. Negli individui immunocompetenti ciò avviene tipicamente senza particolari sintomi clinici: dopo l'infezione iniziale il virus rimane latente sia in forma episomica che integrato nel genoma cellulare a livello del tratto urogenitale e nei linfociti B.

Quasi tutti gli eventi clinici si verificano in condizione di immunodepressione per la riattivazione dell'infezione latente. Le manifestazioni più importanti colpiscono i trapiantati di rene, sottoposti a trattamento immunosoppressivo anti-rigetto, nei quali la nefropatia da Polyomavirus BK (PVN: Polyoma Virus Nephropaty) può portare anche all'insufficienza dell'organo trapiantato (3).

Dopo trapianto, secondo recenti dati di letteratura, il 30-60\% dei riceventi sviluppa una viruria, il 10-20\% una viremia per BK ed il 5-10\% una PVN (1).

Scopo del nostro studio è quello di valutare l'utilità di un test molecolare per il monitoraggio della viruria e della viremia da BKV nei trapiantati renali.

\section{MATERIALI E METODI}

Nel periodo gennaio 2009-giugno 2010 abbiamo effettuato il follow-up dell'eventuale riattivazione di BKV di 29 trapiantati renali (19 maschi e 10 femmine, età media 51 anni).

La terapia immunosoppressiva (Tabella 1) era così articolata: 9 pazienti erano in trattamento con Ciclosporina A (CyA: 4 $\mathrm{mg} / \mathrm{kg} / \mathrm{die}$ con eventuale modulazione sulla base dei livelli plasmatici), Azatioprina (Aza: $1.5 \mathrm{mg} / \mathrm{kg} / \mathrm{die}$ ) e Metilprednisolone (P: $10 \mathrm{mg} / \mathrm{die}) ; 10$ con Tacrolimus (FK506: $0.1 \mathrm{mg} / \mathrm{kg} / \mathrm{bis}$ in die con eventuale modulazione sulla base dei livelli plasmatici) e Mofetil Micofenolato (MMF: 500 mg/bis-ter in die); 7 con FK506, MMF e P ed infine 3 con CyA, P, MMF e FK506.

Lo screening per BKV-DNA è stato condotto mensilmente su sangue e urine con il kit PCR nested della ditta Nanogen Advanced Diagnostic s.r.l. L'estrazione dell'acido nucleico è stata eseguita con il kit Extragen, specifico per i campioni di fluidi non cellulari quali: plasma raccolto in EDTA, siero, urine, liquido cefalorachidiano, liquido amniotico, sovranatante da gargarizzato, da tampone faringeo, ecc.

L'estrazione è stata effettuata seguendo le istruzioni della ditta produttrice: la lisi del campione con un agente caotropico (guanidina cloridrato), un detergente (CTAB) e un agente riducente (2-mercaptoetanolo); dopo precipitazione ad alta temperatura delle proteine e lavaggio con etanolo al $70 \%$, gli acidi nucleici venivano disciolti in acqua ultrapura.

La PCR nested prevede l'esecuzione di due successive reazioni di coamplificazione: una prima specifica per una regione del gene codificante il Large T Antigen (la principale proteina di regolamentazione che si esprime nelle prime fasi del ciclo virale) è condotta nella prima provetta partendo dal DNA estratto dai campioni; una seconda specifica per la stessa regione è condotta nella seconda provetta partendo dal prodotto della prima reazione di amplificazione. La presenza del prodotto specifico della seconda reazione di amplificazione indica la presenza del DNA di BK nel campione di partenza. I campioni positivi sono stati sottoposti a valutazione quantitativa con il kit Real Time PCR della stessa ditta, utilizzato sullo strumento AB 7000 della Applied Biosystem.

Come controllo interno del campione da testare il kit usa il gene della beta-globina e per la determinazione del titolo di DNA devono essere utilizzati gli standard a concentrazione nota forniti nel kit.

Mensilmente i pazienti sono stati seguiti anche sotto l'aspetto della citologia esfoliativa urinaria, che rappresenta un ottimo strumento per individuare precocemente complicanze immunologiche e non immunologiche del trapiantato renale.

Nei pazienti con viruria e/o viremia da BKV è stata effettuata, con la tecnica di Papanicolau, la ricerca urinaria delle decoycells, cellule tubulari con tipici corpi inclusi endonucleari,

\section{RISULTATI}

Diciassette su ventinove pazienti (59\%) sono sempre risultati negativi su sangue e urine (Figura I). Dei rimanenti 12 (Tabella 2), 6 (50\%, di cui 3 in terapia con FK506, MMF e P, 2 con FK506 e MMF e 1 con CyA, Aza e P) hanno evidenziato positività per viruria (media $10^{4} \mathrm{cp} / \mathrm{mL}$ ) e negatività per viremia; 4 (33\%, di cui 3 in terapia con FK506 e MMF ed 1 con CyA, P, MMF e FK506) positività per viremia (media $\left.10^{5} \mathrm{cp} / \mathrm{mL}\right)$ e negatività per viruria ed infine in $2(17 \%$, di cui 1 in terapia con CyA, P, MMF e FK506 ed 1 con FK506 e 
MMF) sono risultate positive sia la viremia che la viruria (entrambe $>10^{6} \mathrm{cp} / \mathrm{mL}$ ) (Tabella 3 ).

Nei pazienti positivi, tenendo conto anche dei valori sostenuti di creatinina (tra 3.9 e $7.8 \mathrm{mg} / \mathrm{dL}$ ) e delle caratteristiche morfologiche del quadro fornito dall'esame citologico urinario, è stata immediatamente rimodulata la terapia immunosoppressiva, evento questo gestito dal clinico sempre con grande cautela; infatti la riduzione della terapia consente di tenere sotto controllo la replicazione virale ma può anche favorire, se non determinare, il rigetto del rene trapiantato. La rimodulazione dei farmaci ha portato, mediamente nell'arco di tre mesi, alla significativa diminuzione della carica virale nel plasma e/o nelle urine, ma mai, finora, alla completa negativizzazione; in due pazienti, malgrado la riduzione della posologia, non è stato riscontrato alcun decremento della replicazione virale, che si è mantenuta costantemente elevata. Dopo consenso informato, tre trapiantati sono stati sottoposti a biopsia renale: un paziente appartenente al gruppo con positività del BKV solo nelle urine, in terapia con FK506, MMF e $\mathrm{P}$, con assenza di decoy-cells, e che comunque manifestava un decadimento rapido della funzionalità del rene trapiantato ed i due pazienti con alte cariche virali nel plasma e nelle urine, sempre con deterioramento renale progressivo.

La biopsia del paziente con sola viruria è risultata negativa per PVN, mentre è risultata positiva (tipiche lesioni tubulo-interstiziali multifocali, specie a carico del tubulo distale, con perdita della tipica polarità della cellula tubulare) nei due pazienti con viremia e viruria costantemente sostenute, malgrado la riduzione della terapia immunosoppressiva. In essi si è verificata, purtroppo, la perdita di funzionalità del rene trapiantato. La positività della ricerca urinaria delle decoy-cells è stata apprezzata in due dei sei pazienti con sola viruria, in due dei quattro pazienti con sola viremia ed in entrambi i pazienti con la contemporanea presenza ad alto titolo di BKV-DNA nel plasma e nelle urine.

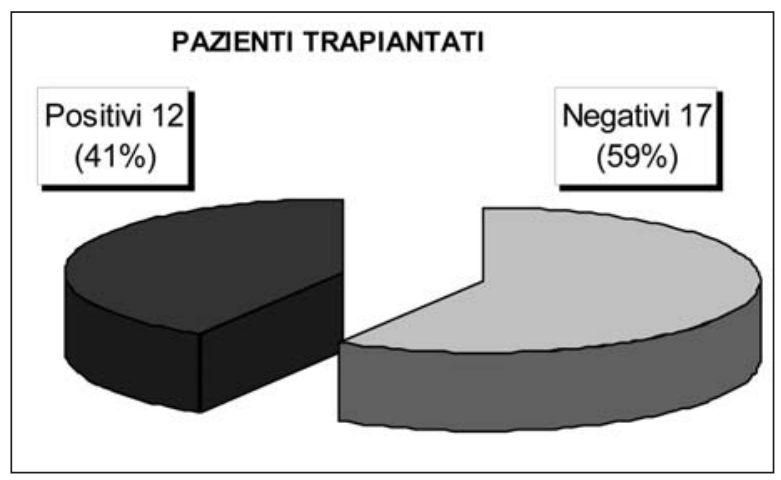

Figura I. Risultati dello screening con BKV PCR nested.

Tabella I. Schema terapeutico nei pazienti trapiantati. TERAPIA IMMUNOSOPPRESSIVA PAZIENTI

\begin{tabular}{lc}
\hline CyA + Aza + P & 9 \\
\hline FK506 + MMF & 10 \\
\hline FK506 + MMF + P & 7 \\
\hline CyA + MMF + P + FK506 & 3 \\
\hline Totale & 29 \\
\hline CyA = Ciclosporina A & \\
Aza = Azatioprina & \\
P $=$ Metilprednisolone & \\
MMF = Mofetil Micofenolato & \\
FK506 = Tacrolimus &
\end{tabular}

Tabella 2. Risultati per BKV-DNA nei I 2 pazienti positivi in base al materiale esaminato.

\begin{tabular}{lccc}
\hline Materiale campioni & \multicolumn{3}{c}{$\begin{array}{c}\text { Pazienti positivi BKV-DNA } \\
(\mathbf{N}=12)\end{array}$} \\
\hline Urine & $\mathbf{6}$ & $\mathbf{4}$ & $\mathbf{2}$ \\
\hline Plasma & + & - & + \\
\hline
\end{tabular}

Tabella 3. Schema terapeutico nei pazienti positivi per BKV. TERAPIA IMMUNOSOPPRESSIVA PAZIENTI

\begin{tabular}{ll}
\hline CyA + Aza + P & I \\
\hline FK506 + MMF & 6 \\
\hline FK506 + MMF + P & 3 \\
\hline CyA + MMF + P + FK506 & I2 \\
\hline Totale & \\
\hline CyA = Ciclosporina A & \\
Aza = Azatioprina & \\
P = Metilprednisolone & \\
MMF = Mofetil Micofenolato & \\
FK506 = Tacrolimus &
\end{tabular}

\section{DISCUSSIONE}

L'escrezione solo urinaria del BKV (50\%) nei pazienti da noi monitorati rientra nel range riportato in letteratura $(30-60 \%)$; sono invece maggiori le percentuali relative all'incidenza di viremia (33\% contro il $10-20 \%)$ e di PVN $(17 \%$ contro il 5$10 \%)(1)$.

I risultati ottenuti, pur non statisticamente significativi, non sembrano indicare una correlazione tra il tipo di trattamento e l'infezione attiva da BKV, come invece è stato riportato in letteratura (2).

La nefropatia interstiziale rappresenta una seria minaccia per l'organo trapiantato e l'individuazione della riattivazione di BKV in seguito al trattamento immunosoppressivo consente, di solito, di poter intervenire prima che essa si instauri.

Non esistendo al momento farmaci antivirali specifici per il BKV, la strategia terapeutica di prima scelta nel controllo della replicazione virale e successiva PVN, è la riduzione della terapia immunosoppressiva.

Nella nostra esperienza la rimodulazione della terapia non si è rivelata sempre efficace nella prevenzione della PVN, a conferma dell'esistenza di altri meccanismi, ancora sconosciuti, probabilmente non solo immunologici, che incidono negativamente sulla funzionalità renale di alcuni soggetti trapiantati.

Diversi Autori, a questo proposito, suggeriscono l'impiego di terapie adiuvanti ancora in corso di validazione (Cidofovir) (4), infusioni di Immunoglobuline o Chinolonici, ma sono ancora necessari studi controllati e randomizzati su popolazioni più vaste per confermarne l'efficacia.

Dal punto di vista strettamente virologico i nostri dati confermano che la contemporanea presenza di viruria e viremia ad alto titolo da Polyomavirus BK costituisce un'evenienza sfavorevole per l'organo trapiantato. Infatti la presenza del virus solo nelle urine è indicativa di una colonizzazione a livello renale non ancora associata a nefropatia.

Per quanto concerne l'utilità della ricerca urinaria delle decoy-cells questa, a nostro avviso, può essere considerata solo un'indagine supplementare, non invasiva, caratterizzata da bassa sensibilità e specificità; non va inoltre sottovalutato l'aspetto relativo al rapido deterioramento delle decoy-cells nel campione biologico, fattori che complessivamente rendono tale ricerca clinicamente poco valida.

La biopsia renale costituisce il gold standard per la diagnosi della PVN.

La PCR nested deve essere comunque considerata un valido strumento diagnostico, specifico e sensibile, per valutare la presenza del virus nelle urine e l'eventuale diffusione sistemica.

Il monitoraggio mensile mediante Real Time PCR quantitativa, che permette di valutare la carica virale rispetto ai livelli basali propri di ogni singolo paziente, rappresenta certamente il miglior metodo non invasivo ed oggettivo per seguire l'infezione da BKV.

Consente inoltre di stabilire il livello di riduzione dell'immunosoppressione al fine di prevenire la progressione del danno renale con conseguente miglioramento della prognosi (5). 


\section{BIBLIOGRAFIA}

1. Beimler J, Sommerer C, Zeier M. The influence of immunosuppression on the development of BK virus nephropathy does it matter? Nephrol Dial Transplant 2007; 22 (8): 66-71.

2. Binet I, Nickeleit V, Hirsch HH, et al. Polyomavirus disease under new immunosuppressive drugs: a cause of renal graft dysfunction and graft loss. Transplantation 1999; 67 (6): 918-22.
3. Hariharan S. BK virus nephritis after renal transplantation. Kidney Int 2006; 69: 655-62

4. Kuypers DR, Vandooren AK, Lerut E, et al. Adjuvant low-dose cidofovir therapy for BK polyomavirus interstitial nephritis in renal transplant recipients. Am J Transplant 2005; 5: 1997-2004.

5. Randhawa P, Ho A, Shapiro R, et al. Correlates of quantitative measurement of BK polyomavirus (BKV) DNA with clinical course of BKV infection in renal transplant recipients. J Clin Microbiol 2004; 42: 1176-80. 